\title{
Simulation of Pavement Random Excitation Based on Harmonic Superposition Method
}

\author{
Kehui Ma*, Yongguo Zhang, Xü Zhen \\ School of Transportation and Vehicle Engineering, \\ Shandong University of Technology, Zibo, Shandong, 255000, China \\ Email: 15969671713@163.com; 17853465307@163.com;xuz1997@126.com \\ *Corresponding author details: Kehui Ma; 15969671713@163.com
}

\begin{abstract}
The road input model is very important in the analysis of vehicle ride comfort and handling stability. Based on the analysis of the relationship between the spatial frequency power spectral density and the time power spectral density of the road, the road signal generation model is established. The simulation is carried out under different vehicle speeds, and the B and C-level random road time excitation signals are generated. The power spectral density is used to compare the simulation results of the model with the road classification standard. The experimental results show that the results are accurate and can provide reliable excitation signals for vehicle control research.
\end{abstract}

Keywords: road roughness; time domain model; random excitation; power spectral density

\section{INTRODUCTION}

As the external input of suspension system, the road excitation model is the research and realization content of vehicle suspension simulation analysis and bench test system. In order to obtain the vibration characteristics of the suspension system, a certain road excitation input is applied to the vehicle suspension system model to make it operate normally. The establishment of a reasonable and accurate road excitation model is of great significance to the analysis of vehicle suspension system dynamics, vehicle ride comfort and road adhesion.

In the past, the research on vehicle ride is based on the assumption of linear system [1]. The frequency domain model of system response can be established directly and conveniently by using the road spectrum input to obtain the results of ride analysis and calculation. It is of great significance in the study of vehicle ride comfort [2] However, the vehicle is a nonlinear vibration system composed of many nonlinear elements. The road model must be described in the time domain for the study of nonlinear system [3]. In the study of vehicle active suspension design and control problems, the time domain analysis method is easier to get a good control law [4]. With the help of time-domain model of excitation, the vehicle ride analysis can be easily carried out in time and frequency domain. Therefore, the time domain irregularity modeling of pavement has caused the research of scholars at home and abroad [5-6].

In the simulation study, if the road excitation signal is not selected properly, the system response will deviate greatly from the actual. In the existing literature, it is difficult to generate time-domain sequence, and almost no verification of the correctness of reconstructed timedomain road is involved, which leads to a significant difference in the time-domain signal of the constructed road.
According to the normalized power spectrum of random pavement, this paper analyzes the relationship between time domain and frequency domain, and finds a reasonable, correct and simple generation method of time domain excitation signal of pavement with the help of MATLAB. It can provide reliable excitation signal for vehicle ride comfort research.

\section{EXPRESSION METHOD OF ROAD ROUGHNESS}

As the input of vehicle vibration, the statistical characteristics of road roughness are usually given in the form of power spectral density in frequency domain. This method is adopted in ISO / TC108 / SC2N67, which gives the spectral density of pavement displacement $G_{q}(n)$ according to the pavement grade in a meaningful range of spatial frequency n. The corresponding values of $G_{q}(n)$ can be taken according to different standards or tests. The power spectral density of road roughness can be fitted by the following formula:

$$
G_{q}(n)=G_{q}\left(n_{0}\right)\left(\frac{n}{n_{0}}\right)^{-\omega}
$$

In equation (1), $n$ is the spatial frequency, and the unit is $\mathrm{m}^{-1}$, which indicates the number of wavelengths contained in each meter of length; $n_{0}$ is the reference spatial frequency, $n_{0}=0.1 \mathrm{~m}^{-1} ; G_{q}\left(n_{0}\right)$ is the road roughness coefficient, and the unit is $\mathrm{m}^{-3}$, which represents the power spectral density of road displacement under the reference spatial frequency; $\omega$ is the frequency index, which is the slope of the diagonal line on the double logarithmic coordinate. It determines the frequency structure of the road power spectral density, 
usually $\omega=2 ; n_{\min }$ and $n_{\max }$ represent the lower limit and upper limit of spatial frequency respectively, usually $n_{\min }=0.011 \mathrm{~m}^{-1}$ and $n_{\max }=2.83 \mathrm{~m}^{-1}$. According to the above documents, the pavement is divided into 8 grades according to the power spectral density, and the geometric average value of each grade of pavement is specified, as shown in TABLE 1.

TABLE 1: Roughness of road surface of 8 ranks standard

\begin{tabular}{|c|c|c|}
\hline $\begin{array}{c}\text { road } \\
\text { surface }\end{array}$ & $\begin{array}{c}G_{q}(n) / \mathrm{m}^{3} \\
\left(n_{0}=0.1 \mathrm{~m}^{-1}\right)\end{array}$ & $\begin{array}{c}\sigma_{q} / \mathrm{m} \\
\left(0.011 \mathrm{~m}^{-1}<\mathrm{n}<2.83 \mathrm{~m}^{-1}\right)\end{array}$ \\
\hline & $\begin{array}{c}\text { geometric } \\
\text { mean }\end{array}$ & geometric mean \\
\hline A & $16 \times 10^{-6}$ & $3.81 \times 10^{-3}$ \\
\hline B & $64 \times 10^{-6}$ & $7.61 \times 10^{-3}$ \\
\hline $\mathrm{C}$ & $256 \times 10^{-6}$ & $15.23 \times 10^{-3}$ \\
\hline $\mathrm{D}$ & $1024 \times 10^{-6}$ & $30.45 \times 10^{-3}$ \\
\hline E & $4096 \times 10^{-6}$ & $60.90 \times 10^{-3}$ \\
\hline $\mathrm{F}$ & $16384 \times 10^{-6}$ & $121.80 \times 10^{-3}$ \\
\hline G & $65536 \times 10^{-6}$ & $243.61 \times 10^{-3}$ \\
\hline $\mathrm{H}$ & $262144 \times 10^{-6}$ & $487.22 \times 10^{-3}$ \\
\hline
\end{tabular}

ESTABLISHMENT OF HARMONIC SUPERPOSITION ALGORITHM MODEL FOR ROAD ROUGHNESS

Since the ride comfort of vehicles is carried out in time domain, the input of road surface should also be expressed in time domain according to the corresponding road surface grade, so the spatial frequency should be converted to the time frequency first. According to the given vehicle speed $u$, the time frequency $f=u n$ can be obtained, and the road power spectral density $G_{q}(n)$ of the spatial frequency $n_{\min }<n<n_{\max }$ can be converted into the road power spectral density $G_{q}(f)$ within the time frequency $f_{\min }<f<f_{\max }$. From equation (1), it can be concluded that:

$$
G_{\mathrm{q}}(f)=G_{\mathrm{q}}\left(n_{0}\right) n_{0}^{2} \frac{u}{f^{2}}
$$

The frequency interval $\left(f_{\min }, f_{\max }\right)$ is discretized into $m$ cells, and the power spectrum at the center frequency $f_{\text {min_k }}$ of the kth cell is used to replace the power spectrum density of the whole cell. The power spectrum of the kth cell is obtained:

$$
w=G_{q}\left(f_{\text {mid_k }_{-}}\right) \cdot \Delta f_{k} \quad(k=1,2, \cdots, m)
$$

In equation (3), $\Delta f_{k}$ is the frequency interval. According to the relationship between the power spectrum and the amplitude spectrum $\left|A^{2}\right|=\omega^{2}$, the amplitude of the irregularity corresponding to each frequency segment can be obtained:

$$
A_{k}=\sqrt{w}=\sqrt{G_{q}\left(f_{\text {mid_k }_{-}}\right) \cdot \Delta f_{k}}
$$

The standard deviation of sine wave $\sqrt{2} A_{k} \cdot \sin \left(2 \pi \cdot f_{\text {mid } k} t+\theta_{k}\right)$ is $A_{k}$, and $m$ sine waves can be superimposed together to obtain the vertical displacement input of random pavement, namely:

$$
x_{\mathrm{r}}(t)=\sum_{k=1}^{m} \sqrt{2} A_{k} \sin \left(2 \pi f_{\text {min }_{-k}} t+\theta_{k}\right)
$$

By introducing $t=\frac{x}{u}$ and $f_{\text {min } \_k_{1}}=u n_{\text {min_k }_{\_}}$into equation (5), we can obtain:

$$
x_{\mathrm{r}}(x)=\sum_{k=1}^{m} \sqrt{2} A_{k} \sin \left(2 \pi n_{\min _{-} k} x+\theta_{k}\right)
$$

In equation (6), $x$ is the longitudinal displacement of the vehicle; $\theta_{k}$ is a random number on $[0,2 \pi]$, which satisfies uniform distribution.

\section{GENERATION AND VERIFICATION ANALYSIS OF RANDOM PAVEMENT DISPLACEMENT}

\section{Random Road Surface Generation of Harmonic Superposition Based On MATLAB}

According to equation (6) and the parameters provided in Table 1, the simulation program is written in MATLAB. In the process of programming, the roughness coefficient $G_{q}\left(n_{0}\right)$ should be set according to the pavement level; The space frequency $n$ is segmented; Set the longitudinal displacement $x$ of the car and segment the road; Speed $u$; In this study, the time domain simulation of B-class road and C-class road under different speeds is carried out. FIGURE 1-4 shows the simulated random road displacement images.

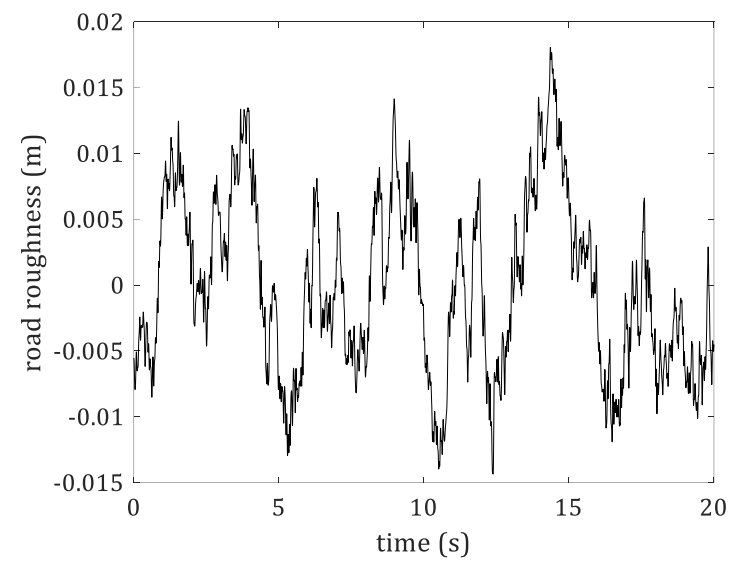

FIGURE 1: The Vehicle Speed is $10 \mathrm{~m} / \mathrm{s}$ Under Class B Road Surface

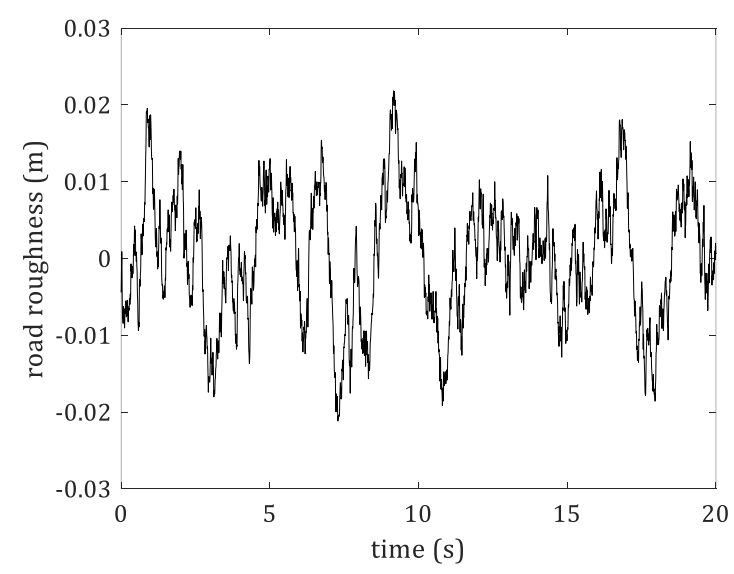

FIGURE 2: The Vehicle Speed is $20 \mathrm{~m} / \mathrm{s}$ Under Class B Road Surface 


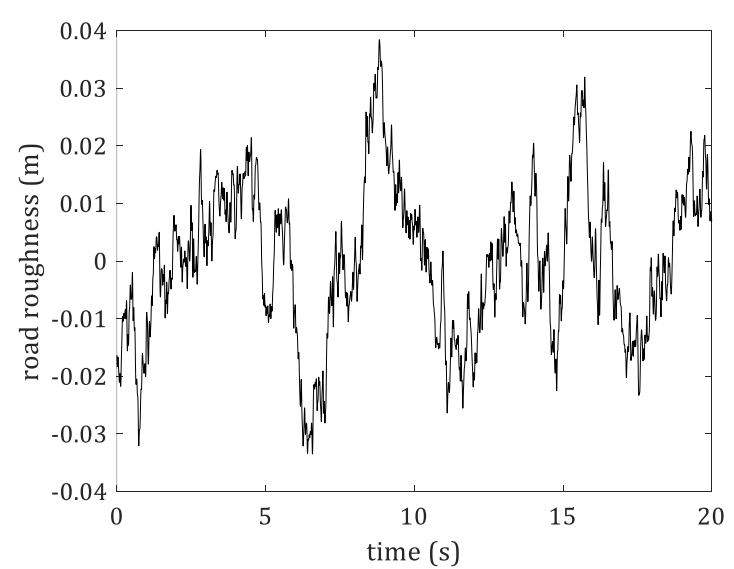

FIGURE 3: The Vehicle Speed is $10 \mathrm{~m} / \mathrm{s}$ Under Class C Road Surface

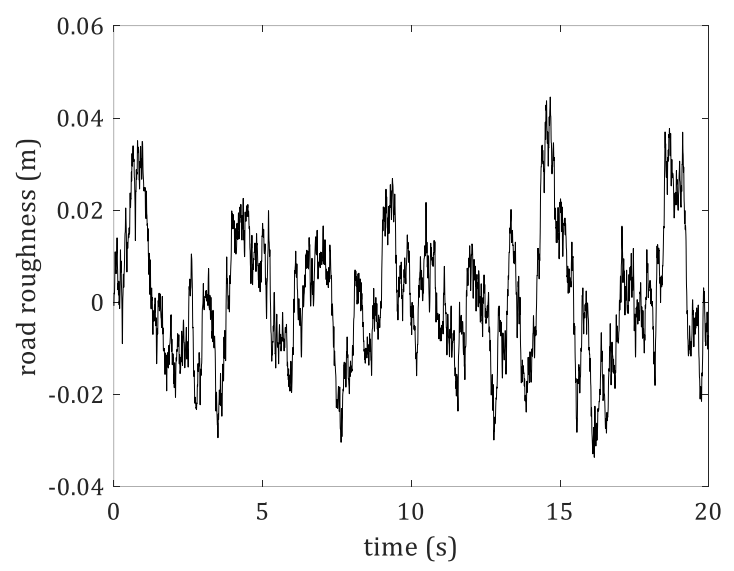

FIGURE 4: The Vehicle Speed is $20 \mathrm{~m} / \mathrm{s}$ Under Class C Road Surface

\section{Validation of random spectral density of pavement}

According to the time domain simulation results of class $\mathrm{B}$ and $\mathrm{C}$ standard pavement, the spectral density contrast curve of random pavement elevation and standard pavement can be obtained, as shown in FIGURE 5. The road displacement input shown in the figure is consistent at any vehicle speed. The red line segment in the figure is the standard power spectrum of road surface, and the black curve is the power spectrum of simulated random road surface.

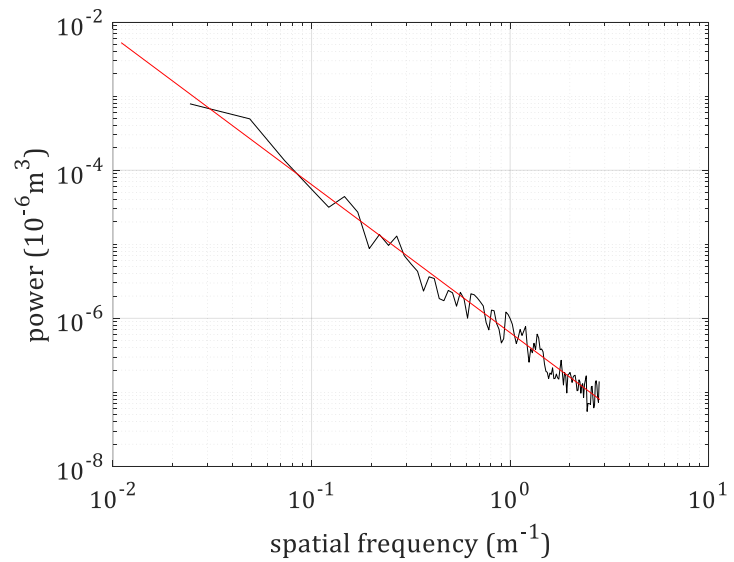

FIGURE 5 : PSD Curve Compare of Gengerated Stochastic Road Surface and Standard Rank Road Surface

(The Vehicle Speed is 10m/s Under Class B Road Surface)

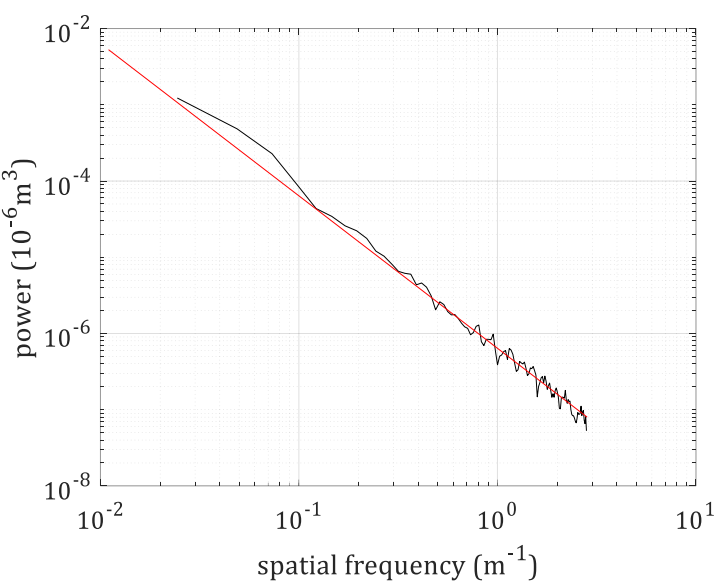

FIGURE 6 : PSD Curve Compare of Gengerated Stochastic Road Surface and Standard Rank Road Surface

(The Vehicle Speed is 20m/s Under Class B Road Surface)

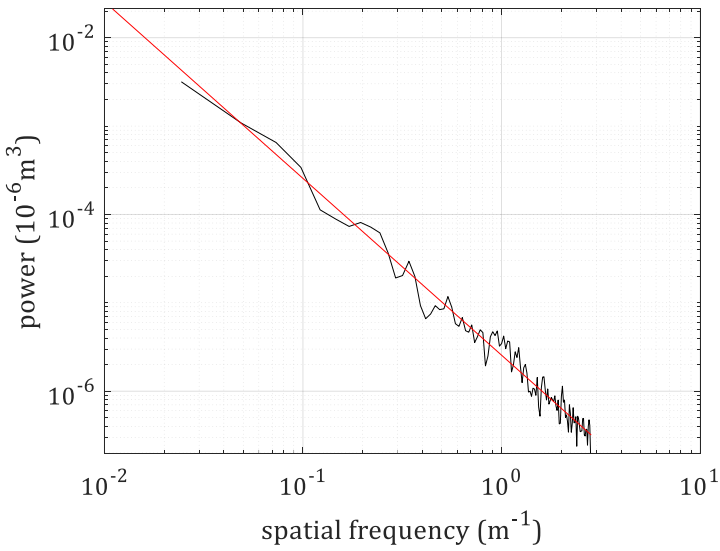

FIGURE 7 : PSD Curve Compare of Gengerated Stochastic Road Surface and Standard Rank Road Surface

(The Vehicle Speed is $10 \mathrm{~m} / \mathrm{s}$ Under Class C Road Surface)

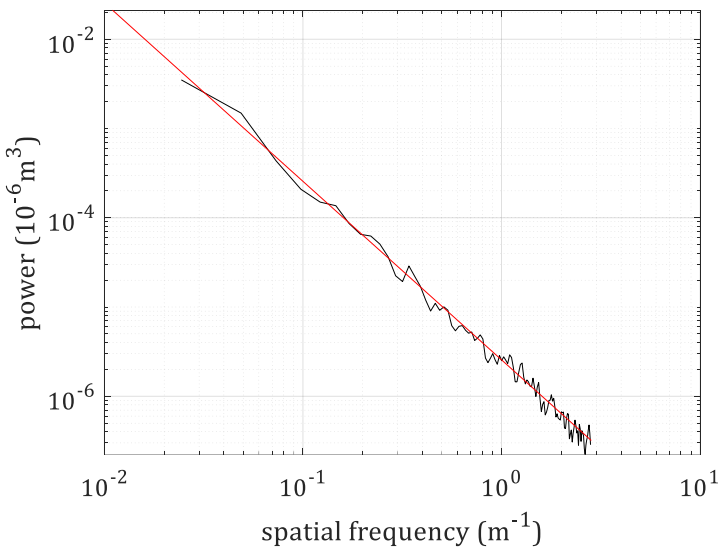

FIGURE 8 : PSD Curve Compare of Gengerated Stochastic Road Surface and Standard Rank Road Surface (The Vehicle Speed is 20m/s Under Class C Road Surface) 


\section{CONCLUSIONS}

By analyzing the relationship between the spatial frequency and the temporal frequency power spectral density of the road, combined with the MATLAB software, a practical road generation simulation model is established.

Through the power spectrum analysis of the simulation results, it can be seen that the road spectrum is in good agreement with the standard road spectrum. The road surface input model of harmonic superposition can directly generate the random elevation according to the standard road surface spatial frequency power spectral density, road surface spatial cut-off frequency, driving speed, etc., which can be used as the input excitation signal for vehicle ride comfort analysis and research. It can be proved that the method in this paper is universal, and can be applied to the generation of excitation signals of different grades of road, providing reliable excitation signals for vehicle control research

\section{REFERENCES}

[1] L. Wei, Y. S. Chen, G. Q. Gong. (1999). Ride performance simulative calculation of vehicle-berthhuman subsystem of road sleeper berth bus. China Journal of Highway and Transport, 12(1), 101-104.

[2] X. H. Tao, X. M. (2003). Huang. Analysis and calculation of frequency domain simulations of dynamic load. J. of HUST: Urban Science Edition, 20(4),47-50.

[3] Kurimoto M, Yoshimura T. (1998). Active Suspension of Cars Using Sliding Mode Controllers (Based on Reduced Models). Int.J. of Vehicle,19(4),402-414.

[4] Y. L. Zhang, Y. F. Zhong. X. M. (2004). Time domain model of road undulation excitation to vehicles. Transactions of the Chinese Society for Agricultural Machinery,35(2), 9-12.

[5] G. W. Tang, X. F. He, Y. F. Yan. (2000). Mathematical model of road roughness in time domain and its simulation. China Journal of Highway and Transport, 13(1), 114-117.

[6] H. Heng, S. F. Lu. (1999). A vehicle time domain model with road input on four wheels. Automotive Engineering, 21(2), 112-117. 